\title{
Heralded single photon sources: a route towards quantum communication technology and photon standards
}

\author{
S.A. Castelletto and R.E. Scholten \\ School of Physics, The University of Melbourne, 3010 Victoria, Australia
}

Received: 15 November 2006 / Accepted: 26 November 2007 Published online: 9 April 2008 - (c) EDP Sciences

\begin{abstract}
Single photon counting, based on single photon sources and detectors, is a key ingredient for certain applications aiming at new quantum information technologies. Quantum cryptography, quantum radiometry, distributed quantum computing, as well as adjacent technologies such as biomedical and astronomical imaging, and low power classical communication also rely on single-photon technology. This paper reviews the present status of single photon sources and related counting measurement techniques, based on correlated (or heralded) photons in parametric down-conversion, and their possible impact on the above mentioned technologies, as well as an assessment for photon standards in the future.
\end{abstract}

PACS. 42.62.Eh Metrological applications; optical frequency synthesizers for precision spectroscopy 03.67.Dd Quantum cryptography - 42.50.Dv Nonclassical states of the electromagnetic field, including entangled photon states; quantum state engineering and measurements - 42.70.Mp Nonlinear optical crystals

\section{Introduction}

The advent of quantum information and computation science has placed stringent demands on optical sources, namely that sources produce single photons either ondemand or heralded, and has also brought into sharper focus the need for better photon-counting detectors [1]. Quantum communication and quantum computation applications place difficult design requirements on the generation, manipulation, processing and detection of single photons [2]. Quantum cryptography [3] would particularly benefit from improved sources and detectors, for example Quantum Key Distribution (QKD), which is currently constrained by low key rate production. When a message is encoded in one degree of freedom of a photon, it cannot be cloned by an eavesdropper without introducing errors. While not optimal, single photons may be useful for cryptography even if they are created at random times. However, some protocols require single photons that are produced at a particular time, i.e. on demand. A single photon source that periodically outputs only one photon with a high probability seems to be essential for linear optics quantum computing (LOQC). The Knill-LaflammeMilburn [2] LOQC approach assumes the availability of single photon sources and detectors, and exploits the photon bunching that occurs in quantum interference between pure state single photons. High visibility in interference between photons from multiple sources is crucial, which means that precise timing of the photons and precise control of the modal structure of the generated photons is required. However, only approximations of such a source are available. Moreover, lack of high brightness single photon sources, and imperfect detector characteristics such as detection efficiency, dark count rate, timing jitter, and dead-time, significantly limit the key rate production in QKD [4]. To meet the demands for higher-rate secret key production and pseudo deterministic pure single photon state sources, the quantum information community is presently engaged in a number of efforts aimed at improving QKD, including optimizing the quantum channels for minimum loss $[5,6]$ (low loss optical storage and fast optical switches), improving detector efficiency $[2,7,8]$, reducing detector timing jitter [9], reducing detector deadtime [10], and single-mode single-photon source engineering $[11-17]$.

In the area of metrology, more specifically radiometry, high-speed photon detection capability could allow the realization of a very bright single-photon source as a photon standard, that in the long term could bring a new radiometric standard yielding a "quantum candela" [18], i.e. a redefinition of the candela based on a countable photon source. A photon standard linked to the definition of the candela could have a remarkable impact in traditional detection applications such as medical diagnosis, and bioluminescence, chemical, and material analysis [19-22], because the photometric unit would be directly linked to biological measurements.

Until the emergence of quantum optics, the "singlephoton regime" was attained by strongly attenuating a laser beam to ensure that the probability of having more than one photon became negligible. However, such attenuated photon sources differ from "true" single 
photons because the vacuum probability is much higher than the probability of detecting a photon, with predominantly a "no-photon" regime and occasional detection of a photon, while the probability of having two photons is never zero.

Single photon sources are presently realized by many technologies, for example nonlinear optics, new photonic materials (photonic fibers), single isolated quantum emitters in an atomic system, and semiconductor devices at the nanostructure level, as well as impurities in diamonds [23]. In general single-photon sources fall into two categories: isolated quantum systems, and two-photon emitters. The first type relies on the fact that a single isolated quantum system can emit only one photon each time it is excited. The difficulties are in obtaining efficient excitation, efficient output collection, and good isolation of individual systems. This review does not deal with these sources, referring instead to already existing reviews on the matter $[23,24]$.

Photons generated with different approaches can present practical properties that have to be analyzed individually to determine their effectiveness for each particular application. Standardized measurement methods are needed to determine which application is effectively addressed by each source. Various attempts at evaluating and comparing the performance of each source have been made, but because in each case the achievements are still at an investigation level, there are not yet definitive answers.

Here we focus on the type of light source which emits two photons at a time, also called a bi-photon field, often referred to as a "heralded" single photon source. A biphoton field can be generated via nonlinear optical interaction in a $\chi^{(2)}$ non-linear material, i.e. parametric downconversion (PDC) [25]. This two-photon light has a key role in applications such as quantum radiometry, guaranteeing a tool for direct photon counting detector calibration without any external standard [26,27], and/or it results in a more convenient single photon source than an attenuated laser $[28,29]$. Other metrological applications of bi-photon fields, where the main interest is in their entanglement property, are also potentially important; for example positioning and clock synchronization [30], quantum imaging [31] and quantum lithography [32]. Worth mentioning is also the application of high intensity PDC fields for quantum information and quantum imaging. In fact while quantum information has been initially developed for discrete quantum variables, which is in practise realized with single photon states, much attention has been recently devoted to the continuous variable (CV) regime and to multiphoton states of light [33]. In this regime quantum teleportation and generation of multimode entanglement have been proven, as well as quantum imaging. Bright and stable entanglement sources based on optical parametric processes have been successful to implement quantum imaging protocols, but the characterization of CV entanglement remains challenging. Recently, measurement of quantum correlations in mesoscopic twinbeams, generation of mesoscopic single beam nonclassi- cal states, have been shown [34]. Sub-shot-noise photon number correlation has been proven in multi-mode pulsed PDC by measuring not only the correlation function of the twin-beams, which alone is not sufficient to state the presence of quantum correlation, but also the distribution of the difference photon counts, which better identify quantum versus classical correlations [34]. The key challenges in this measurement are to balance the detections losses of the two arms, to select twin coherence areas, and to estimate the overall detection efficiencies, in order to evaluate the degree of quantum correlation of the source. These measurements are promising for the conditional generation of sub-Poissonian light at the mesoscopic level which is the $\mathrm{CV}$ counterpart of the conditional preparation of a single photon state.

While PDC is relatively easy, its use in metrology applications as well as in quantum information is not straightforward. Some schemes of how to adopt PDC [35-37] in the realization of heralded single photon source are reported in Section 2, addressing the main difficulty of having an almost deterministic production of single photons from an actual random process of photon production. In the same section the problem of multiphoton production is also analyzed with possible solutions. PDC can be generated either in bulk or in periodically poled crystals. In both cases the emission is multi-mode and broadband, and post-selection techniques must be adopted and the pump photons must be forced into a definite and controlled spatial mode, to optimize the mode matching of the emitted photons. In Section 3 we review the theory of PDC state decomposition in multi and single modes, considering the inseparability between spatial and spectral degrees of freedom of the states. In Section 4, a review of production of heralded photon sources in bulk nonlinear crystal is provided, discussing various experiments [38-45], both with CW and pulsed pump configurations. In Section 5 we will review attempts to generate heralded single photons in periodically poled crystals [46-50]. Section 6 reviews the use of waveguided periodically poled crystals. Waveguides have been used instead of post-selection, maintaining a high level of photon creation. In this case correlated photons are generated in single modes directly, and the usual problem associated with the correlation between spatial and spectral degrees of freedom is solved. Further development of this single photon source could include modal engineering of the photon states and mode matching into singlemode fibers, given that the waveguide exhibits accurate modal control [11-13,51-55], considering a much more precise analysis by Schmidt decomposition of spatio-temporal state amplitude [56]. Sections 7 and 8 deal with a general overview of practical heralded photon sources for quantum communication and radiometry.

\section{Heralded single photon source realization}

A heralded single photon source is based on a source of two photons at a time, whereby the detection of one photon indicates the existence of the second photon, allowing the 


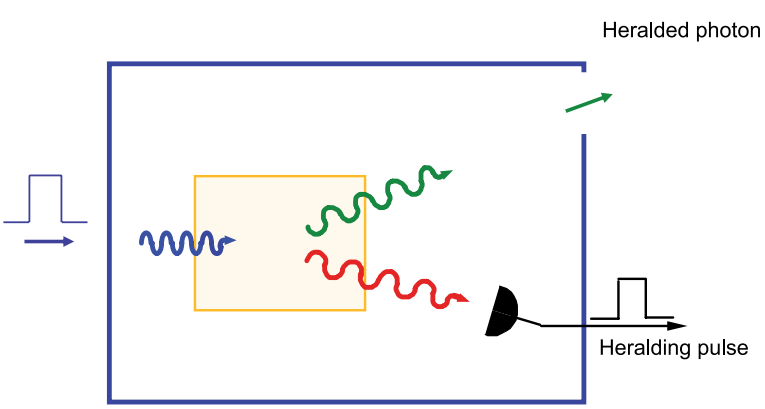

Fig. 1. Principle of a heralded photon source, generated by a pump laser pulse in a nonlinear optical crystal yielding twin photons, correlated in time, energy and polarization. One photon is detected and its presence heralds the second photon.

second photon to be manipulated and delivered to where it is needed. The original idea was firstly sketched by Klyshko [25], as illustrated by Figure 1.

There are two main problems with this type of source. One is the random production in time of single photons, i.e. it is not an on-demand source. The other is that the photons are not necessarily single, particularly in a high gain regime.

The first problem is partially overcome by using a pulsed pump that allows the production of twin-photons only during the pump pulse duration. The heralded photon can then be needed at a specific time, that is ondemand, and synchronized to other optical systems processing the information carried by the photon. Side effects of very short pump pulses (fs) inside a nonlinear crystal will be analyzed in Section 4. The first approach used to realize a single heralded photon source on-demand was reported in reference [35], where a storage loop was used for the heralded photons, released later in time when necessary.

The second problem to be solved is multiphoton production. Multi-pair production per pulse is regulated by Poisson statistics, therefore the probability of multiple pairs scales with the square of the probability of producing a single pair. This means that more than one photon can be produced, for a given detected photon. It is possible to keep this probability low in a regime of low pair production rate, but this implies also a low heralding photon rate, which means a slow system. A possible solution was proposed in reference [36] and later implemented in a simpler scheme [57]. By using a multiplexed heralding source (Fig. 2b) it is possible to maintain a low pair production in each down-converter and therefore a low probability of multi-pair production, but the pool of down-converters yields an overall higher and tailored probability of having a final heralded single photon, even in the presence of a low multiphoton probability. A similar result can be obtained also by using a pump with a high repetition rate (several $\mathrm{MHz}$ ) (time multiplexed) or recycling a pump pulse within a single down-converter [37], when a combination of the scheme (a) and (b) of Figure 2 is used.

Reference [58] is the first experimental realization and characterization of an asynchronous heralded single pho- (a)

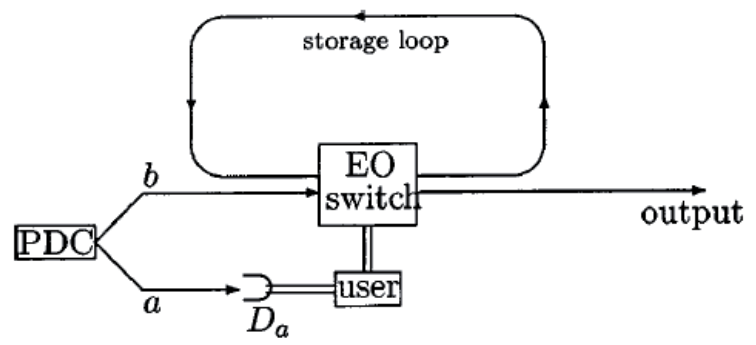

(b)

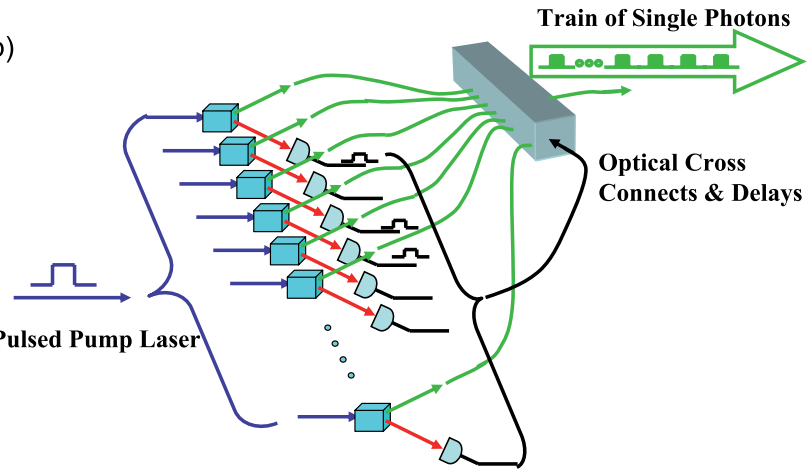

Fig. 2. (a) Heralded single photon source on-demand [35], where the heralded photon, when announced by its twin, is switched in a storage loop and then it can be switched out at a later time according to user need. This scheme can be used in a variety of quantum information processing applications. (b) A multiplexed single photon source on-demand uses a pool of down-converters, pumped by the same laser pulse, to generate twin photons [36]. The heralding detector activates a fast optical switch to allow the corresponding correlated photon to be released in a train of single photons.

ton source based on PDC with photons at $1550 \mathrm{~nm}$, heralded as being inside a single-mode fiber with more than $60 \%$ probability and with the heralding arm at $810 \mathrm{~nm}$. Here the source is called asynchronous because the heralding photons are not synchronized with a periodic clock, the PDC pump being continuous. The heralded photon output port is connected to a Hanbury-Brown-Twiss setup to characterize the relevant parameters $\mathrm{P}(1)$ (the probability of having exactly 1 photon) and $\mathrm{P}(2)$ (the probability of having more than 1 photon). $\mathrm{P}(2)$ is reduced by a factor up to more than 500 compared to Poissonian light sources. Schemes to convert this source into a synchronous system were described, including a pulsed laser to pump the nonlinear crystal and a switched storage delay line.

Another obstacle with heralded single photon sources is the difficulty to isolate and collect single modes correlated one by one. To address this problem the community is trying several solutions: deep investigation of the modal emission of those sources; use and investigation of new more promising materials from emerging photonics technologies; design of specific nonlinear optical material embedded in waveguides; and photonic materials more appropriate to the application. 


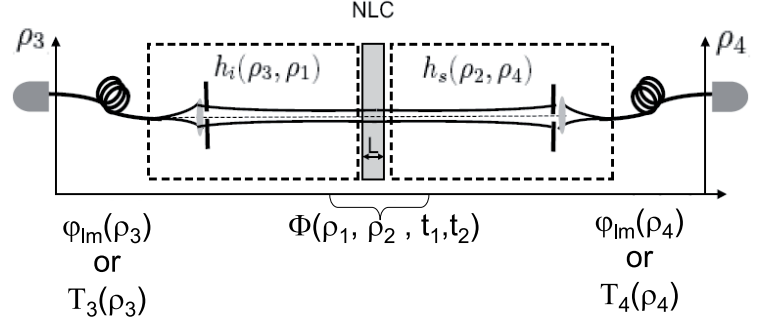

Fig. 3. Unfolded picture of the heralding channel (acting as a source) of a PDC (one photon of the PDC arm, collecting optics, and a detector). The nonlinear crystal NLC is of length $L$. The object (on the left) can be represented by either an incoherent or coherent source depending on whether the heralding beam is prepared by an aperture (or a multi-mode fiber) or by a single-mode fiber. On the right hand side of the scheme the heralded photon is collected and detected, and the coincidences are counted between the heralding and heralded photon detection.

\section{Heralded single photons via PDC: Theory of mode preparation}

PDC is known to produce a quantum state of light with a two-photon field description [25], that exhibits a quantum behavior only when measured in coincidence. Given the multi-mode photon production and intrinsic spectral and spatial quantum correlation of the modes of the two photons (the two-photons are described by an entangled state), the heralded photon is often in an incoherent, mixed state. In other words, if only one photon of the pair is measured, the source exhibits purely thermal statistical behavior, given by intrinsic multi-mode photon production. This is because the heralding channel normally consists of an integrated time detection in combination with passive spatial and spectral filtering. We can nevertheless introduce a certain degree of coherence. By properly measuring one of the photons, we prepare the other photon in a specific state $[59,60]$. The prepared state will be pure only if the heralding PDC photon is projected into a single mode.

Two different approaches can be used to prepare heralded single photons, distinguished by how the heralding and heralded photons are collected. One uses single-mode fiber, while the second uses a multi-mode spatial filter. In quantum information processing applications [29], pulse-pumped PDC has been employed, followed by narrow-band spectral filtering (for temporal mode definition) $[61,62]$, and coupling the photons into single-mode fibers (for spatial mode definition). The pulsed operation guarantees a higher gain in photon production, but requires precise modeling of the source spatial and spectral correlation interplay. Multimode preparation has been employed for the calibration of single-photon detectors [27]. Let us consider the generic scheme of principle in Figure 3 to define the heralded photon mode preparation. We consider the two-photon state approximated as [63]

$$
|\psi\rangle=\int d \rho_{1} d \rho_{2} d t_{1} d t_{2} \Phi\left(\rho_{1}, \rho_{2}, t_{1}, t_{2}\right)\left|1_{\rho_{2}, t_{2}}\right\rangle\left|1_{\rho_{1}, t_{1}}\right\rangle
$$

where $\rho_{1,2}$ represent the transverse positions of the two output photons at the times $t_{1,2}, \Phi\left(\rho_{1}, \rho_{2}, t_{1}, t_{2}\right)$ is the bi-photon field, which contains the correlation between the two photons in the spatio-temporal degree of freedom, thus yielding an entangled photon pair. The biphoton field results from the pump envelope and the phase-matching function defined by the specific downconversion configuration. $\left|1_{\rho_{2}, t_{2}}\right\rangle,\left|1_{\rho_{1}, t_{1}}\right\rangle$ represent the single photon modes. PDC is realized for two kinds of phasematching conditions, known as Type I and II. Type I corresponds to an extraordinary beam downconverting into two ordinary beams, therefore the photon pairs present the same polarization, and the PDC is characterized by one conical emission around the pump direction. Type II means one of the emitted PDC photons has ordinary polarization, while its twin has extraordinary polarization. As a result, each photon of a pair is emitted into separate, nonconcentric and orthogonally polarized output cones of light. Only type II realizes polarization entangled photons. Heralded photon sources can be realized with both Type I and II.

\subsection{Single mode preparation}

Envisioning the optics setup for a PDC process as the unfolded scheme of Figure (3) [64], the source (heralding arm) is described by the propagation of a coherent mode defined by $\varphi_{l m}\left(\rho_{3}\right)$ through an optical element with impulse response function $h_{i}\left(\rho_{3}, \rho_{1}\right)$, through the nonlinear crystal where the mode is transformed according to the function $\Phi\left(\rho_{1}, \rho_{2}, t_{1}, t_{2}\right)$, and collected eventually after propagating with $h_{s}\left(\rho_{2}, \rho_{4}\right)$ by the field $\varphi_{l m}\left(\rho_{4}\right)$ (this last corresponding to the heralded photon detection). The subscripts $i, s$ stand for idler and signal, as historically indicated the twin photons in PDC.

The bi-photon field $\Phi\left(\rho_{1}, \rho_{2}, t_{1}, t_{2}\right)$ is analytic only at the first-order approximation of the transverse wavevectors and depends on the Fourier transform of the pump angular distribution and the phase-matching function (as in Ref. [65]).

The coincident detections at positions 3 and $4, \mathcal{C}_{34}$, are given by the overlap between the PDC field and both of the preparation and collection modes, while the single counts $\mathcal{C}_{3}$ and $\mathcal{C}_{4}$ measure individually the overlap between the biphoton field and each of the preparing or collecting modes:

$$
\begin{aligned}
\mathcal{C}_{34}= & \int d t_{1} d t_{2} \mid \int d \rho_{1} d \rho_{2} d \rho_{3} d \rho_{4} \Phi\left(\rho_{1}, \rho_{2}, t_{1}, t_{2}\right) \\
& \times\left. h_{i}\left(\rho_{1}, \rho_{3}\right) h_{s}\left(\rho_{2}, \rho_{4}\right) \varphi_{l^{\prime} m^{\prime}}^{*}\left(\rho_{4}\right) \varphi_{l m}^{*}\left(\rho_{3}\right)\right|^{2} \\
\mathcal{C}_{3,4}= & \int d t_{1} d t_{2} d \rho_{4,3} \mid \int d \rho_{1} d \rho_{2} d \rho_{3,4} \\
& \times\left.\Phi\left(\rho_{1}, \rho_{2}, t_{1}, t_{2}\right) h_{i}\left(\rho_{1}, \rho_{3}\right) h_{s}\left(\rho_{2}, \rho_{4}\right) \varphi_{l m}^{*}\left(\rho_{3,4}\right)\right|^{2} .
\end{aligned}
$$




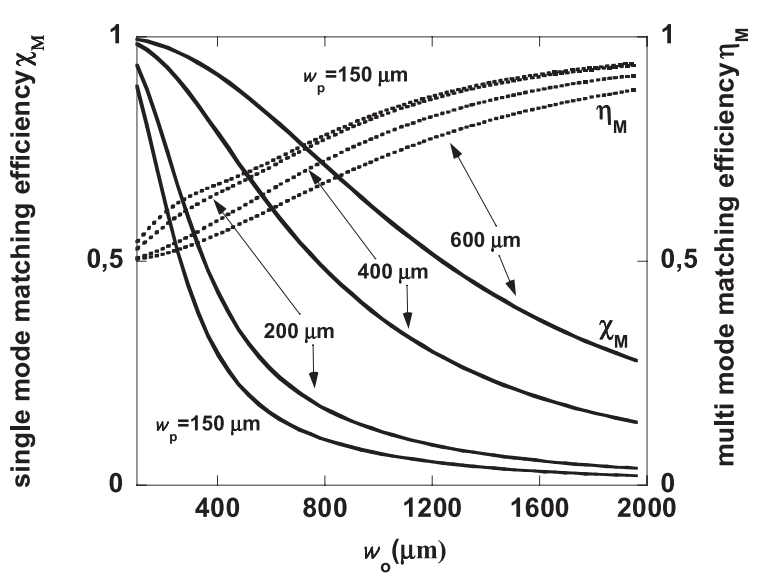

Fig. 4. Plots of single and multi-mode matching efficiency, $\chi_{M}$ (solid) and $\eta_{M}$ (dash) and various pump waists, versus the waist of the preparing and collecting mode, $w_{o}$.

The spatial coherence of the single guided modes in the heralded and heralding arms should ultimately match the overall spatial coherence of the two-photon states.

To quantify the purity of the prepared state, it is crucial to properly define and measure the efficiency of that preparation, which is obtained from the ratio between the coincident detections and the single counts in the heralding arm.

\subsection{Multi-mode preparation}

The multi-mode preparation consists of the collapse of the PDC wave function over the spatial distribution of multimode fibers or spatial filters (apertures). Referring to the unfolded scheme of Figure 3, the source is incoherent with finite transverse distribution $\mathcal{T}_{3}\left(\rho_{3}\right)$. The collecting modes are then defined by the spatial filter and given by $\mathcal{T}_{4}\left(\rho_{4}\right)$. The coincidences measured at positions 3 and 4 are then $C_{34}$, and $C_{3}$ and $C_{4}$ are the single counts:

$$
\begin{aligned}
C_{34}= & \int d t_{1} d t_{2} d \rho_{3} d \rho_{4} \mathcal{T}_{4}\left(\rho_{4}\right) \mathcal{T}_{3}\left(\rho_{3}\right) \\
& \times\left|\int d \rho_{1} d \rho_{2} \Phi\left(\rho_{1}, t_{1}, \rho_{2}, t_{2}\right) h_{i}\left(\rho_{1}, \rho_{3}\right) h_{s}\left(\rho_{2}, \rho_{4}\right)\right|^{2} \\
C_{3,4}= & \int d t_{1} d t_{2} d \rho_{4} d \rho_{3} \mathcal{T}_{3,4}\left(\rho_{3,4}\right) \\
& \left|d \rho_{1} d \rho_{2} \Phi\left(\rho_{1}, t_{1}, \rho_{2}, t_{2}\right) h_{i}\left(\rho_{1}, \rho_{3}\right) h_{s}\left(\rho_{2}, \rho_{4}\right)\right|^{2} .
\end{aligned}
$$

As Figure 4 demonstrates, the predictions made for single and multi-mode preparation efficiencies $\left(\chi_{M}\right.$ and $\eta_{M}$, respectively) yield different results. We remind that single and multi-mode preparation efficiencies are given by $[60,66]$

$$
\chi_{M}=\frac{\mathcal{C}_{34}}{\sqrt{\mathcal{C}_{3} \mathcal{C}_{4}}}, \eta_{M}=\frac{C_{34}}{\sqrt{C_{3} C_{4}}},
$$

and they depend on the pump transverse waist $\left(w_{p}\right)$ and on the collection/preparation fiber-defined mode at

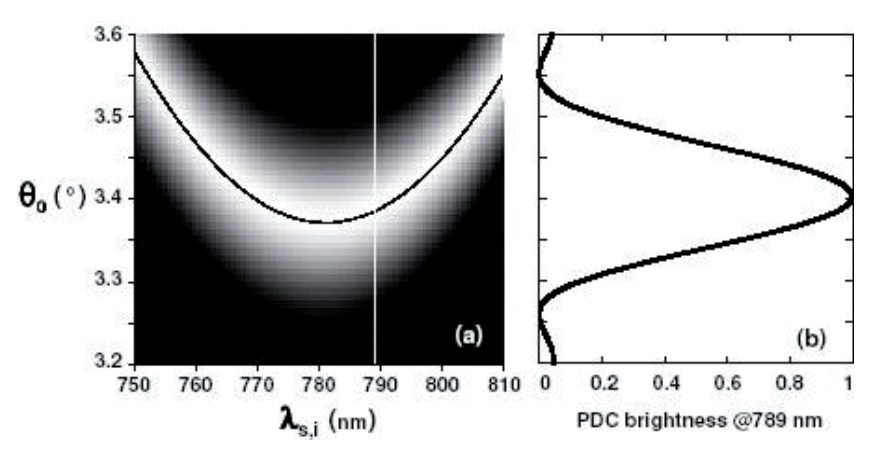

Fig. 5. (a) Calculated [68] external angular PDC emission of $\mathrm{LiIO}_{3}$ at a phase-matching angle of $44.15^{\circ}$, including the longitudinal mismatch and the pump transverse shape (Gaussian with a $260 \mu \mathrm{m}$ pump waist, crystal length $L=5 \mathrm{~mm}$ ). The black line is the central emission angle of PDC for a $395 \mathrm{~nm}$ CW pump beam. (b) Vertical slice of (a) at a wavelength of $789 \mathrm{~nm}$.

the crystal, $w_{o}$. When $w_{p} \ll w_{o}, \eta_{M}$ asymptotically approaches 1 , while $\chi_{M}$ goes to 0 ; in the opposite condition $\left(w_{p} \gg w_{o}\right), \eta_{M} \rightarrow \frac{1}{2}$ and $\chi_{M} \rightarrow 1$ [66]. For multi-mode preparation, with a fixed pump waist $w_{p}$, the maximum mode-matching efficiency is obtained when $w_{o}$ is large, i.e. all the pumped crystal volume is in a region of unit efficiency of the spatial filter system. For singlemode preparation, one of the fibers acts as a source of a single-mode beam that propagates through a spatial filter (in this case the pumped crystal volume) to the other fiber. The maximum mode-matching efficiency is achieved with a large pump waist, with respect to the preparation and collection beam waist at the crystal. Similar results have been reported in reference [67], where the analysis of distinguishability of the polarized entangled photons is performed versus the pump focusing in the cases of a multi-mode or single mode channel collection. The results show that in multi-mode collection, a collimated pump is preferable both in terms of detection of single and coincidence events, while the single mode operation requires a focused pump to allow higher coincidence rate.

\subsection{Spectral mode preparation}

Even in the continuous wave $(\mathrm{CW})$ case for type I phase matching, spectral mode selection is crucial. This is easily understood from the PDC phase-matching function in Figure 5. The phase-matching function modulates the PDC output brightness for each wavelength of PDC emission, and it depends on the longitudinal mismatch between the pump, signal and idler wavevectors, on the crystal length, on the transverse pump field distribution, and eventually on the pump pulse distribution. Figure 5 shows the calculated output angle $\theta$ of the down-converted photons as a function of wavelength [68]. The thickness of the curve is due to the finite dimension transverse pump beam and the finite crystal length, when longitudinal mismatch is included. Calculating the PDC emission for a range of pump beam diameters and crystal lengths demonstrates that the 
fuzziness or spread of the emission angle is due to these effects. For example, a larger pump beam waist gives reduced fuzziness, while a longer crystal, where longitudinal phase-mismatch is present, decreases the spread around the central emission angle.

In pulsed pump operation the spectral and spatial selection are more interconnected. Pulsed pumping was first included in the calculation of the bi-photon field in reference [42], where an approximated analytical result for the coincidence probability and the spectral coincidence in type I phase-matching is provided and compared with the exact numerical calculation. The conclusion was that the analytical solution holds only for short crystals and even in this case a departure from the exact solution is evident. In reference [41] the effect of the pulsed pump beam on the PDC spectrum width is reported only from the phenomenological point of view, to justify experimental results. While in reference [44] a theoretical analytic approach to predict the spectral emission of the PDC coincidence and to optimize the spectral and spatial mode selection is adopted and compared with the experiments.

In pulsed pump operation the bi-photon field depends also on the time Fourier transform of the pump spectral distribution. In this calculation, spectral selection considerations have to be included to properly predict the coincidence spectrum and the actual matching/collection efficiency. The fiber spectral selection is deduced from the PDC angular central emission curve. The angular selection of a fiber-defined single mode beam, considered as a spatial filter, around the central emission angle of the PDC is obtained from $\theta_{o}\left(\lambda_{s, i}\right)$ (see Fig. 5). The geometric collection selected spectral width component at the full width half maximum (FWHM) by the fiber, $\Delta_{1,2}$, is approximately given by the FWHM angular collection $\Delta \theta_{1,2}$ of the fiber times the slope of the spectral/angular spread of the PDC, around the central wavelength $\lambda_{o}$, i.e.

$$
\Delta_{1,2}=\Delta \theta_{1,2}\left[\frac{\partial \theta_{o}(\lambda)}{\partial \lambda}\right]_{\lambda_{o}}^{-1}
$$

where $\Delta \theta_{1,2}=\sqrt{2 \ln 2} \frac{\lambda}{\pi w_{o, 1,2}}$, and $w_{o, 1,2}$ are the fiber waists at the crystal. Spectral selection is achieved by an interference filter, and/or by single spatial mode collection. A single mode fiber (SMF) acts as a spectral filter with a Gaussian spectral distribution given by

$$
\widetilde{I}_{\nu_{s, i}}\left(\nu_{s, i}\right)=\exp \left[-\frac{2 \ln 2}{\Delta_{1,2}^{2}} \nu_{s, i}^{2}\right]
$$

with $\Delta_{1,2}$ being the FWHM of the spectral distribution, $\nu_{i, s, p}=\omega_{\mathrm{i}, \mathrm{s}, \mathrm{p}}-\Omega_{\mathrm{i}, \mathrm{s}, \mathrm{p}}$ the displacement of idler, signal and pump frequency from the central frequencies indicated by $\Omega_{\mathrm{i}, \mathrm{s}, \mathrm{p}}$. The coincidence spectrum is determined by Fourier transforming only the transverse spatial variables of $\Phi\left(\rho_{1}, \rho_{2}, t_{1}, t_{2}\right)$ and the resulting frequency-dependent bi-photon field is calculated by integrating over the transverse spatial variables according to

$$
\Phi\left(\nu_{s}, \nu_{i}\right)=\int \mathrm{d} \rho_{1} \mathrm{~d} \rho_{\mathbf{2}} \Phi\left(\rho_{1}, \rho_{2}, \nu_{s}, \nu_{i}\right) \varphi_{10}^{*}\left(\rho_{1}\right) \varphi_{20}^{*}\left(\rho_{2}\right) .
$$

The coincidence spectrum for the degenerate case $\left(\Omega_{s}=\right.$ $\left.\Omega_{i}\right)$ is

$\mathcal{C}_{34}\left(\nu_{i}\right)=\int \mathrm{d} \nu_{s}\left|\Phi\left(\nu_{s}, \nu_{i}\right)\right|^{2} \widetilde{I}_{\nu_{i}}\left(\nu_{i}\right) \widetilde{I}_{\nu_{s}}\left(\nu_{s}\right)=\int \mathrm{d} \nu_{s} \mathcal{C}\left(\nu_{i}, \nu_{s}\right)$.

We explicitly write $\mathcal{C}\left(\nu_{i}, \nu_{s}\right)$ as

$$
\begin{aligned}
\mathcal{C}\left(\nu_{i}, \nu_{s}\right) \propto & \left|\widetilde{E}_{\nu_{p}}\left[\left(\nu_{s}+\nu_{i}\right)\left(1+\frac{16 \ln 2 \zeta^{2}}{T_{p}^{2}}\right)^{1 / 2}\right]\right|^{2} \\
& \times \widetilde{I}_{\nu_{i}}\left(\nu_{i}\right) \widetilde{I}_{\nu_{s}}\left(\nu_{s}\right) \\
& \times\left|\operatorname{Erf}\left[\gamma+\zeta\left(\nu_{s}+\nu_{i}\right)\right]-\operatorname{Erf}\left[\zeta\left(\nu_{s}+\nu_{i}\right)\right]\right|^{2}
\end{aligned}
$$

where $\widetilde{E}_{\nu_{p}}$ is the envelope of the pump spectral distribution, with $T_{p}$ its temporal width. From equation (6) the correlation between the spectral components $\nu_{i, s}$ and the transverse parameters $w_{o, p}$ is contained in $\zeta$ and $\gamma$ in the Erf terms, which is relevant to both the pump pulse envelope and the pump transverse distribution. Note that, while for $w_{p}$ large, the first factor contributes to a widened coincidence spectrum, the Erf terms instead reduce the overall coincidence spectrum. For pump waists bigger than $300 \mu \mathrm{m}$, the overall effect is a very slight dependence of the coincidence spectrum on the pump waist. However, when we sharply focus the pump beam waist to the order of $30 \mu \mathrm{m}$, we expect a broader coincidence spectrum. This means that for small pump waist, the correlation is strong and the spectral and transverse components are not separable. Similar results were obtained in reference [42], even for an approximated solution.

\section{Heralded single photons in bulk $\chi^{(2)}$ crystals}

As an example, we describe a fiber-coupling strategy, implementing the above theory (preparing the heralding photon and collecting the heralded photon in single spatial modes) as in Figure 6. In both channels we define our single modes by backward propagating and imaging the single-mode fibers onto the PDC crystal. This strategy was first adopted in reference [38] where the idea was to match the angular distribution of the PDC light for a given spectral bandwidth to the angular width of the spatial mode collected into single-mode fiber. In the original paper the aim was to optimize the collection of correlated photons to maximize the coincidence visibility. The main result was to observe a higher matching/collection efficiency when the single collected mode waists were about the same as the pump waist, for a short crystal; the effect of the transverse spatial distribution of the pump was not observed. A more rigorous study of the coupling efficiency of SMFs with PDC was done in reference [39], which included crystal parameters as well as optical propagation parameters. In particular, it was demonstrated how the pump beam waist, crystal length, optical system magnification and the fiber mode waist must obey a precise joint relation in order to ensure high coupling efficiency. Theoretical predictions have been verified experimentally using 
Table 1. Comparison of recent results in term of $P(1)$, the probability of finding a heralded photon given that a heralding photon has been detected.

\begin{tabular}{|c|c|c|c|c|}
\hline PDC process & $P(1)$ & Heralded bandwidth (nm) & Comments & Reference \\
\hline $532 \mapsto 810+1550$ PPKTP WG & $60 \%$ & 0.44 & CW, Ppump $<1 \mathrm{~mW}$ & {$[52]$} \\
\hline $710 \mapsto 1310+1550$ PPLN WG & $38 \%$ & not reported & CW, Ppump $<1 \mathrm{~mW}$ & {$[54,69]$} \\
\hline $532 \mapsto 810+1550 \mathrm{KNbO}_{3}$ & $60 \%$ & 6.9 & CW, Ppump $\sim$ tens of $\mathrm{mW}$ & {$[58]$} \\
\hline $400 \mapsto 800+800$ PPKTP WG & $85 \%$ & 50 & pulsed, Ppump $<1 \mathrm{~mW}$ & [13] \\
\hline $390 \mapsto 780+780$ BBO bulk & $83 \%$ & 9 & pulsed, Ppump $\sim 80 \mathrm{~mW}$ & [41] \\
\hline $394.5 \mapsto 789+791 \mathrm{LiIO}_{3}$ bulk & $70 \%$ & 5 & pulsed, Ppump $100 \mathrm{~mW}$ & {$[44]$} \\
\hline $532 \mapsto 810+1550$ PPLN bulk & $48 \%, 90 \%$ & $4,0.2$ & CW, Ppump $\sim 100 \mathrm{~mW}$ & {$[49]$} \\
\hline $390 \mapsto 521+1550$ BBO bulk & $18.7 \%$ & 18 & pulsed, Ppump $\sim 200 \mathrm{~mW}$ & [70] \\
\hline
\end{tabular}

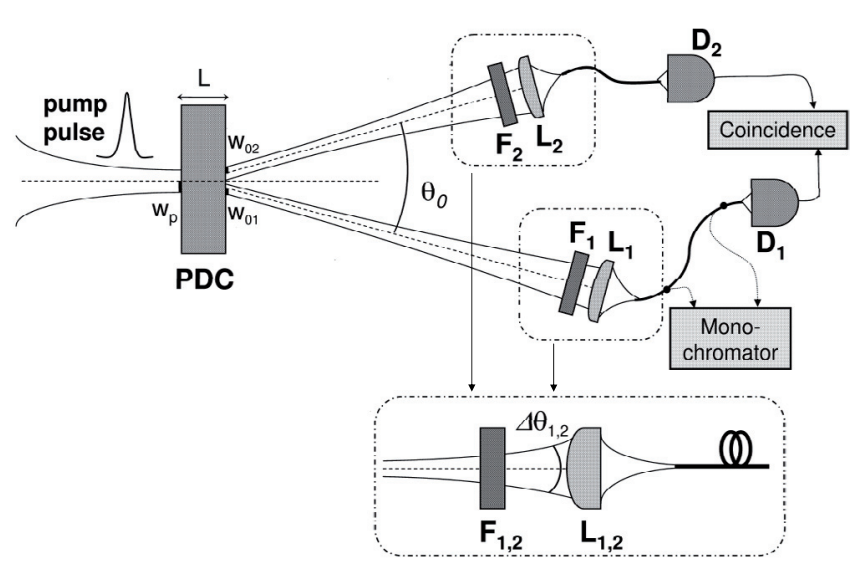

Fig. 6. Setup used to herald single-photons from pulsed PDC in a $5 \mathrm{~mm} \mathrm{LiIO}{ }_{3}$ pumped by frequency-doubled Ti-Sapphire laser (repetition rate of $76 \mathrm{MHz}$ at $394.5 \mathrm{~nm}$, pulse duration $150 \mathrm{fs}$ ) [44]. $\mathrm{L}_{1}$ and $\mathrm{L}_{2}$ are aspheric lenses or $20 \mathrm{X}$ microscope objectives, filters $\mathrm{F}_{1,2}$ select heralding and heralded photons. Direction 1 was the heralding channel. A monochromator was inserted in the heralding channel fiber link to measure the coincidence spectral distribution.

femtosecond-pumped Type II PDC in nonlinear crystals of variable thickness [39]. A more complete theory of the mode matching efficiency for the general case in CW pump operation was then provided [40].

In reference [41] the heralding efficiency was defined as the probability that the twin photon is actually present in its fiber, given the detection of one PDC photon. It was measured in the case of a bulk-crystal type-I PDC source pumped by a mode-locked laser. The effect of the pumping bandwidth of several nanometers was considered by calculating the PDC tuning curves for several different values of pump wavelength. Heralded down-converted photons present a raw bandwidth much greater than $100 \mathrm{~nm}$. Such broad bandwidth corresponds to heralded photons whose coherence length is too short to be used in typical multiphoton interference experiments. A spectral filter to reduce the bandwidth of the accepted heralding photons causes the bandwidth of the heralded photons to also be reduced.

In reference [44] the pulsed pump effect on PDC was modeled as in reference [42], but also included an experimental test. A formula for the absolute heralding efficiency was given as well as theoretical and experimental results comparable to the results reported in reference [41]. The experimental heralding efficiency (or mode preparation efficiency) was obtained by measuring the raw detection efficiency, $\chi_{D}$ and then correcting for the optical losses in the heralded-photon arm, $\tau_{o p t}$, and Single Mode Fiber-lens transmittance, $\tau_{S M F-l e n s}$,

$$
\chi_{p}=\frac{\chi_{D}}{\eta_{\text {det }} \cdot \tau_{o p t} \cdot \tau_{S M F-l e n s}} .
$$

$\chi_{D}$ was simply defined as the coincidence counting rate, $R_{c}$, divided by the single photon detection rate, $R_{1}$ (trigger), $\chi_{D}=R_{c} / R_{1}$. Loss in the heralded-photon path includes the quantum efficiency $\eta_{\text {det }}$ of the heralded detector. Note that $\chi_{p}$ is an estimate of $P(1)$. Heralding efficiencies of some of the reviewed experiments are listed in Table 1.

It has been shown that spatial overlap alone is not enough when we have pulsed pumping and that attention has to be paid to define spectrally the mode preparation efficiency. In reference [44] the PDC source was obtained by a high repetition rate short pulse laser. Two small diametrically opposed regions of the emitted cone were coupled into single-mode fibers using three optical configurations. The beam waists at the crystal were very precisely measured, while the Gaussian pump beam was focused at the crystal with $260 \mu \mathrm{m}$ waist. The coincidence spectrum was analyzed when only the fibers act as spectral filters and adding a spectral filter $\mathrm{F}_{1}$ centered at $789.3 \mathrm{~nm}$ with FWHM of $5 \mathrm{~nm}$ in the heralding channel (trigger) to obtain the best heralding efficiency. $F_{1}$ reduced the bandwidth of the accepted trigger photons along with the bandwidth of the heralded photons and its choice tailors the spectral properties of the heralded photons. Figure 7 shows the effects of restricting the accepted heralded photon bandwidth to $5 \mathrm{~nm}$ around the central photon frequency, versus the case where only the raw bandwidths selected by the two fibers are used, for three different spectral widths given by different lens configurations $L_{1,2}$. It was shown that, even in the absence of the interference filter, the overall coincidence bandwidth is reduced with respect to the single fiber selection spectral bandwidths. This is a signature of entanglement between transverse and spectral components for a weakly focussed pump. To restrict the coincidence bandwidth, a narrow filter on the heralding arm is necessary. To obtain the highest heralding efficiency, the spatial modes, spatially wider than the 


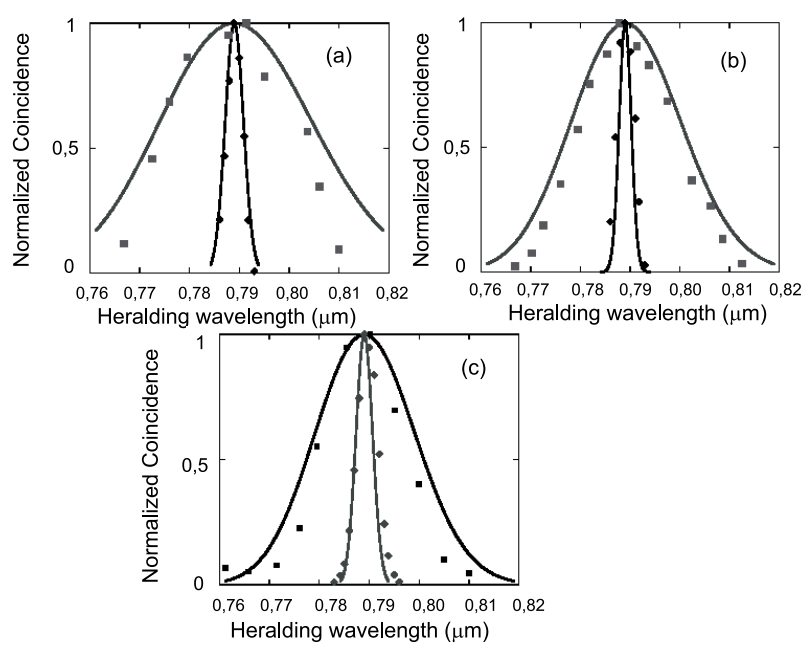

Fig. 7. Spectral scan of the normalized coincidence rates performed with a monochromator in the heralding fiber path. Solid lines are theoretical curves of equation (5). Different lens configurations correspond to (a), (b) and (c) scans. The narrower scans were taken with a $5 \mathrm{~nm}$ FWHM interference filter on the heralding arm (Ref. [44]).

spectral modes selected by the fiber, have to be matched while the heralded arm bandwidth must be significantly wider than that of the trigger-photon filter $\mathrm{F}_{1}$. The theoretical predictions match quite well with the experimental results.

A heralded single photon source in bulk crystal with a pulsed pump was also demonstrated in [70]. Here the heralded photons were at $1550 \mathrm{~nm}$, more suited for fiberbased QKD applications, while the heralding photons were in the visible. The spatial mode matching of PDC with single mode fibers was performed as in the previous references, while there is no mention of spectral matching. The final heralding efficiency results were lower than expected, possibly because of the non-degenerate configuration (see Tab. 1).

\section{Heralded single photons in periodically poled crystals}

Periodic poling of nonlinear crystal materials is a technique for obtaining quasi-phase matching (QPM) of nonlinear interactions. It involves a process which generates a periodic reversal of the domain orientation in a nonlinear crystal, so that the sign of the nonlinear coefficient also changes. QPM often makes it possible to use the same polarization direction for all interacting waves, and this often corresponds to using a stronger element of the nonlinear tensor. Widely used in applications such as optical parametric oscillators and second harmonic generation, it had also been applied in the generation of correlated photon pairs in PDC and lately in applications such as heralded single photon sources. The advantage of using periodically poled (PP) crystals is a greater efficiency of the correlated photon production. In a periodically poled mate-

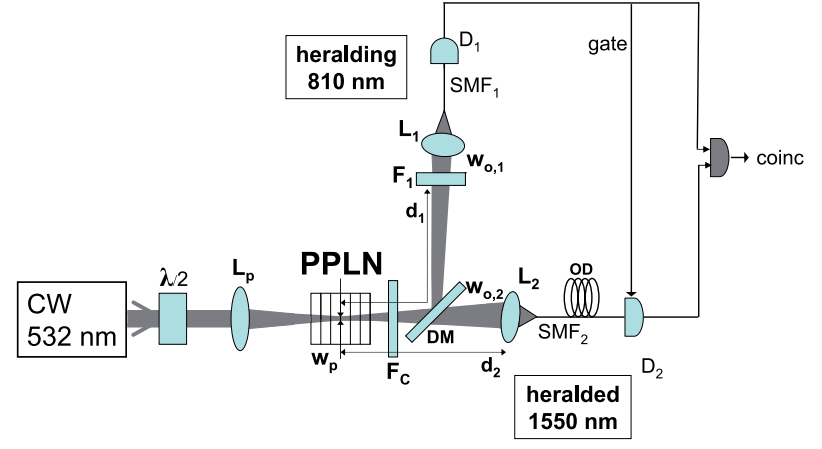

Fig. 8. Setup to herald single photons from PDC in a $5 \mathrm{~mm}$ PPLN crystal, $w_{o, 1,2}$ waists at the crystal for the 2 beams 1 and $2[49]$.

rial a higher $\mathrm{PDC}$ pair production efficiency is guaranteed for QPM, obtainable in each period of the material at a given temperature, making it possible to utilize the largest value of the effective nonlinear coefficient. Production of photon pairs from PP crystals was initially attempted to produce photons pairs at the telecom wavelength for optical communication purposes [47]. Broadband twin photons at $1550 \mathrm{~nm}$ were generated, efficiently coupled into a single-mode fiber. Lately $[46,48] \mathrm{PP}$ crystals were used to produce efficient sources of frequency non-degenerate, polarization-entangled photon pairs. In PP crystals the theory for mode preparation is equivalent to the bulk except that there is no birefringence, because of the non critical phase-matching. Reference [45] is the first work addressing the mode preparation efficiency in collinear emission in QPM bulk crystals. This work is a theoretical and experimental investigation of the emission characteristics and of the flux of photon pairs. It was shown that, by careful design, one can attain high coupling efficiencies, maximized by varying the focusing of the pump mode and the fiber-matched modes. Numerical calculations suggest coupling efficiencies above $93 \%$ at optimal focusing, independent on the length of the crystal. It was pointed out also in this case the connection between spatial and spectral modes, given by the phase-matching. An estimate of the frequency filtering by a single mode fiber was found to be $\propto 1 / L$. This sets how narrow the bandwidth of an additional spectral filter has to be in order to have the highest coupling efficiency. Moreover the interesting result is that longer crystals narrow the bandwidth as well as increase the photon flux coupled to the fibers. This suggest better engineering of the source by having longer crystals, rather than reducing the bandwidth by an additional spectral filter, to reach the highest coupling efficiency.

Similar experimental results, even if the theoretical approach was completely different, were obtained in reference [49], with an experimental configuration as in Figure 8 . Here a periodically poled lithium niobate (PPLN) was used, pumped by a CW laser at $532 \mathrm{~nm}$ to produce $810 \mathrm{~nm}$ and $1550 \mathrm{~nm}$ photon pairs in a slightly noncollinear geometry (PDC photons at $810 \mathrm{~nm}$ and $1550 \mathrm{~nm}$ at external angles of $1^{\circ}$ and $2^{\circ}$ ). The pump was focused and the $1550 \mathrm{~nm}$ corresponds to the heralded photon arm. 
Photons were collected by SMFs. The InGaAs detector $\left(D_{2}\right)$ was operating in gated mode, switched by the heralding Si detector $\left(D_{1}\right)$. While the heralded arm is spectrally selected only by the fiber, the large improvement achieved by narrowing the heralding bandwidth indicates that spectral mode matching is more critical than spatial mode matching (the only spatial requirement is that $w_{o, 2} \cong w_{p}$, $\left.w_{o, 1}>w_{p}\right)$. It has been possible using a very narrow spectral selection obtained with a monochromator, to reach $\chi_{p}$ close to $90 \%$, after correction for the estimated optical losses. However, because this was at the expense of the signal to noise ratio, the uncertainty was quite large. Therefore, in spite of originally higher photon pair production and even when used with a visible heralding arm (therefore in a non degenerate configuration), because of strong spectral post-selection to optimize the heralding efficiency, PPLN crystals yield eventually a poor heralded photon source.

\section{Heralded single photons in periodically poled waveguide crystals}

A common approach to guarantee good mode preparation between PDC photons generated both in bulk and PP-crystals uses strong spatial and spectral filtering. The cost of this, however, is a prohibitive reduction of the generation rate of usable single photon wavepackets. As a consequence, the fidelity of the prepared heralded photons is limited due to vacuum contributions. To eliminate these and to ensure, for example, successful gate operations in LOQC, post-selecting by coincidence measurements is inevitable. Waveguided PDC is one route to accomplish both, to decorrelate the spatial from spectral degrees of freedom ones and to eliminate at the same time the spatial correlations between the PDC photons. Waveguides (WGs) have been used instead of post-selection with an increased source brightness and heralding efficiencies limited only by detection losses. The main issue with WGs is however the separation between the two beams of correlated photons.

Reference [11] reports the generation of pairs of coincident photons at $854 \mathrm{~nm}$ from a PPLN waveguide $1 \mathrm{~cm}$ long. The waveguide structure acts as a spatial filter to select a single transverse mode of PDC light, and without requiring narrow band filters or spatial filters, the photon bandwidth was less than $1 \mathrm{~nm}$, for a CW pump source. The correlated photons were not however separated.

Reference [12] reports the observation of correlated photon pairs generated by PDC of a 400-nm pump pulse in a QPM KTP nonlinear waveguide with the ratio of coincidence to single-photon count rates observed near $800 \mathrm{~nm}$ exceeding 18\%. Correlated photons were generated spectrally broadband $(100 \mathrm{~nm})$ and by a Type I nonlinear interaction. References [54,69] report on a guided wave heralded photon source based on the creation of nondegenerate photon pairs by PDC in PPLN waveguide using Type I interaction. Using the signal photon at $1310 \mathrm{~nm}$ as a trigger, a gated detection process permitted the announcement of the arrival of a single photon at $1550 \mathrm{~nm}$ at the output of a single mode optical fiber with a probability of $38 \%$.

In reference [13] single photons based on waveguided PDC were produced with the highest so far obtained heralding efficiency, 85\%, and extraordinarily high detection rates, due to the efficient pair splitting at the output of the WG. This was realized through a nonlinear interaction producing orthogonally polarized (and therefore spatially separable) photon pairs, using a Type-II PDC interaction in a QPM KTP waveguide. The output of a mode-locked titanium sapphire laser (100 fs pulse duration, $87 \mathrm{MHz}$ repetition rate) with power of $15 \mu \mathrm{W}$ was focused using a $10 \mathrm{X}$ microscope objective into the input face of a $12 \mathrm{~mm}$ long KTP waveguide formed by ion exchange. The photon pairs were split by a polarizing beam splitter and the horizontally polarized signal mode was coupled by a multimode fiber. Despite of small $\mathrm{d}_{24}$ element (responsible for the Type II interaction) of KTP, considerably smaller in magnitude than those elements yielding TypeI PDC $\left(\mathrm{d}_{33}\right.$ and $\left.\mathrm{d}_{31}\right)$, it is possible to obtain a remarkably high production rate of Type-II PDC photon pairs since the modes are confined within the cross section of the waveguide throughout the entire length of the structure. For a single photon source at telecom wavelength, the best configuration is however with the heralding photons in the visible, where single photon detectors are more efficient [52].

As a final comment on the high efficiency of photon pairs in WGs, we underline the difficulty to compare their results on an absolute scale, determined in terms of photon pair production efficiency (PPPE).

A validation test of the absolute measurement of PPPE in WG was performed (Ref. [71]). PDC was based on a WG realized with PPLN crystal, degenerate at $1570 \mathrm{~nm}$. Figure 9 shows the experimental setup used to perform the PPPE measurement based on coincidence detection of PDC photon pairs. Because of the presence of the BS this is not a heralded photon source, therefore the heralding channel is here just as a trigger. Because of high dark counts and high after-pulsing (i.e. the tendency of a detector to send a false detection signal immediately after a real detection event), detector $\mathrm{APD}_{1}$ was gated by $\mathrm{APD}_{2}$ (trigger detector), thus maintaining a sufficiently low (some $\mathrm{kHz}$ ) gating frequency.

From the measured coincidences and trigger detector single counts, the rate of produced pairs is given by

$$
\Lambda_{c}=\frac{2 p_{c}^{\text {true }}}{p_{w / 2}^{0} \eta_{1} \eta_{2} \tau_{1} \tau_{2} T},
$$

where $p_{c}^{\text {true }}$ is the probability of true coincidences, and $p_{w / 2}^{0}$ is the probability of the gated detector not firing in the first half of the coincidence window $w$ (Fig. 10). $\eta_{1,2}$ are the detection efficiencies of the two APDs, $T$ the measurement time, and $\tau_{1,2}$ are the overall optical transmittance for the photons in each channel. According to equation $(8)$, the measured photon pair rate was $(1.35 \pm 0.32)$ $10^{7}$ pairs $/ \mathrm{s}$ for a guided pump power of $(33 \pm 4) \mu \mathrm{W}$. The PPPE was compared with an analogue independent measurement, traced back to a national radiometric standard, 


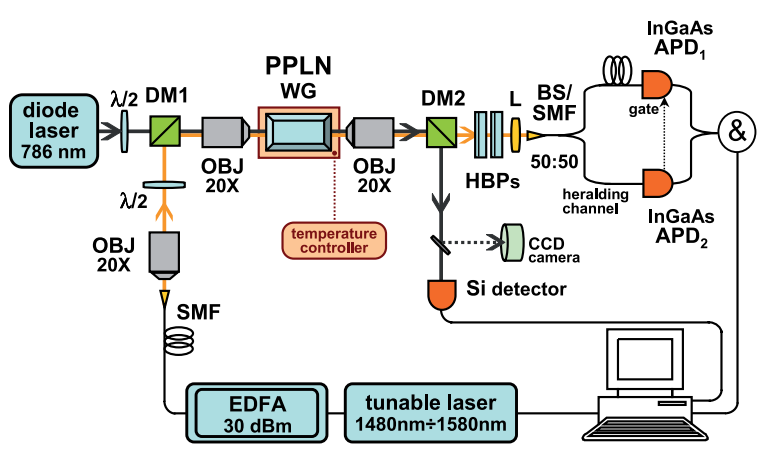

Fig. 9. Setup used to generate single photons at $1572 \mathrm{~nm}$ from a CW pump diode laser at $786 \mathrm{~nm}$, focused onto the WG (annealed proton exchange, $17 \mathrm{~mm}$ long) by a microscope objective (OBJ) [71]. PDC photons were collected and collimated by OBJs, coupled to SMFs by a single aspheric lens (L). A dichroic mirror (DM2) and long-pass filters (HBP) separated the pump from PDC photons. The pump reflected by the dichroic mirror was directed toward a calibrated silicon detector. A seed beam (tunable laser) at telecom wavelength is coupled in the guide to simplify the alignment and the maximization of the collection of the PDC photons into the SMF. PDC photons were routed to a fiber integrated 50:50 beam splitter (BS/SMF), and sent to single photon counters $\left(\mathrm{APD}_{1,2}\right)$, both InGaAs avalanche photo-diodes operating in gated mode.

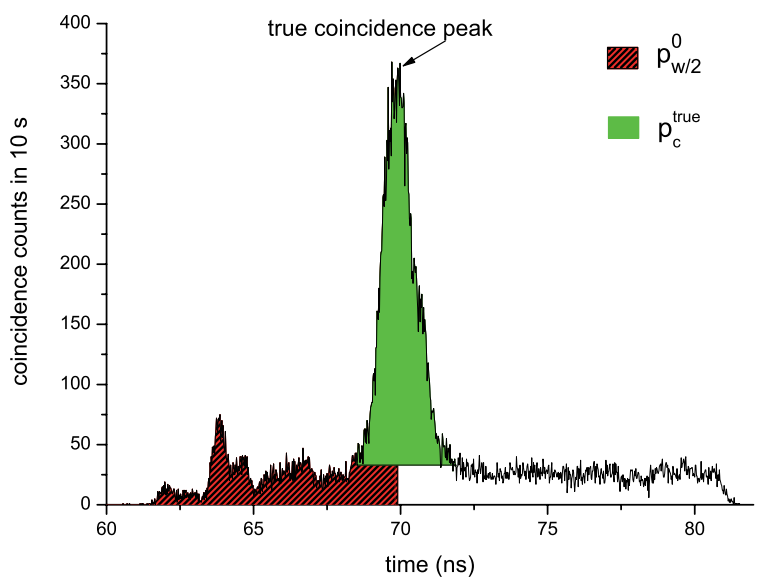

Fig. 10. Coincidence histogram in the experimental condition of coincidence window $w=20 \mathrm{~ns}$, trigger gate $100 \mathrm{kHz}$ (Ref. [71]).

of the PDC spectral power, with good agreement within the uncertainties of the two methods, thus validating the estimate of PPPE in the photon counting regime.

In reference [56] a theoretical study of the conditional state preparation of single photon wavepackets based on PDC pumped by ultrafast pulses is reported. The study investigates the premise given above, that a two photon state lacking correlations between the signal and idler photons with unobserved degrees of freedom, is a basic requirement for ideal single photon preparation. Assuming that spatial correlations can be independently eliminated (for example with a WG), two techniques to obtain spectrally factorizable two-photon states are discussed. Firstly, it was shown that if an additional condition on the two photon state is fulfilled, the group velocity matching condition derived by Keller et al. [72] can yield a state in which both the joint temporal intensity, and the joint spectral intensity are factorable. The latter is crucial to guarantee full factorizability in two photon states designed for conditional preparation of single photons. Secondly, a novel technique is introduced in which the group velocity mismatch between the pump and downconverted photons, responsible for the mixedness of the prepared single photons, can be controlled by using a sequence of crystals alternated with birefringent spacers exhibiting a dispersion which compensates that of the crystal in a specific manner. This represents a powerful technique in which spectrally decorrelated states, as well as a more general class of spectrally engineered two photon states, can be obtained simply by varying the relative thicknesses of the crystal and spacer used.

\section{Practical Heralded single photon sources for quantum communication}

As summarized in the previous sections, a number of different approaches for building such a practical single photon source for quantum communication and QKD have been proposed. References [54,69] provide a comparison analysis of the pros and cons of these approaches. While the final verdict is yet to be made, it appears that at the current state of technological development, PDC is the most practical way to generate single photons on demand, but no practical device has been built to date. A few parameters are of key importance for a practical heralded single photon source for fiber-based QKD: first of all, the heralded photons must travel a significant distance in the optical fibers and this limits the choice of the pump laser and of the nonlinear medium used for parametric process at the telecom windows. Secondly, since the maximum communication link distance is usually limited by the signal-to-noise ratio (SNR) at the receiving side (called Bob), the transmitting side (called Alice) must be able to maximize the collection efficiency of the heralding photons and Alice's detector should have minimal dark count probability, limiting the possible wavelength range of the heralding photons in the visible. Also the erroneous count rate of Bob's detectors must be minimized. This means that no other photons (except for those heralded) should be sent from Alice. Also, since Bob's detectors are typically run in gated mode and their dark count probability is proportional to the duration of the gate, the heralded photon arrival time should be very well known in order to minimize the detector active time and dark count. Chromatic dispersion effects that increase the uncertainty in the photon arrival time and result in longer required gate durations depend on the optical length of the photon travel. Moreover the temporal spreading of the photon wave packet is proportional to its optical bandwidth; hence the latter must be limited in order to decrease Bob's detector gate duration and noise level. Finally, to be practical, a heralded single photon source should be pumped 
by a low power pump laser and must operate reasonably close to room temperature.

A number of groups throughout the world have made experimental advances towards building a practical heralded single photon source. Both bulk medium as well as WG approaches have been tried; recent results are summarized in Table 1, which is far from exhaustive, given the many attempts in this direction. As already mentioned the advantage of using a WG is the disentanglement between the spectral and spatial correlation, which is present in bulk or PPLN crystals, even if the heralding efficiency is still limited by the losses in the waveguide and the non-ideal mode matching of the waveguide modes and the single modes of the fiber. The coupling efficiency from non-circular planar waveguides into optical fibres remains poor unless significant effort is made to engineer the guided mode. Moreover the degenerate case is not favorable in terms of spectral emission, which is too broad, while a non-degenerate configuration is more suited to the applications described here.

A step forward in the direction of practical heralded photon sources for quantum communication has been the development of microstructured and photonic crystal fibres (PCFs), enabling the engineering of profoundly different optical properties to conventional optical fibres. PCFs with very small solid cores can have zero dispersion wavelength in the visible and near infra-red region of the spectrum, while the very small guided mode area leads to extremely high optical intensity, giving rise to ultrahigh optical nonlinearities. For example, even though silica glass does not possess an intrinsically high nonlinearity, the small core size in PCFs leads to strong mode confinement and useful nonlinearities even for low pump power. The effects are thus amplified and give us an effective $\chi^{(3)}$ better than $\chi^{(2)}$ in nonlinear crystals. PCFs allow the generation of photon pairs via four-wave mixing, in which two pump photons are converted into a signal and an idler photon. A review of a heralded photon source based on PCF is given in [73]. In one of the latest experiments [74], non-classical interference of heralded photons from two independent PCFs has been demonstrated in two regimes: a low pump power regime $(1 \mathrm{~mW}$ per fibre), where a high non-classical visibility of $88 \%$ was observed, and a higher pump power regime $(8 \mathrm{~mW}$ per fibre) where the non-classical visibility reached $95 \%$, after subtracting the contribution from multiphoton production. The theoretical maximum for the visibility was found to be $97 \%$. Compared to a PDC light source, an all-fiber two photon light source has advantages in high spectral brightness, broad bandwidth, single spatial mode, low noise background, low collection loss, compact physical size, low pump power and compatibility with an optical network. With appropriate dispersion control, fiber-based two-photon light sources can cover the spectral range from UV to the infrared, all in a single spatial mode, meeting wavelength requirements for quantum communication and possibly other applications.

\section{Practical Heralded single photon sources for radiometry}

The application of bi-photon fields to radiometry developed in the 1980s when Klyshko [75] put forward the first scheme to calibrate single photon detectors without using any reference standard. This proposal was followed by several attempts $[76,77]$ that were essentially proofs of principle and feasibility tests of the technique for real metrology applications. A survey of the pros and cons of this source as a real photon standard are reported in reference [78] and discussion of the possible accuracy and uncertainty achievable with this technique is provided in reference [79]. Even if from the point of view of modal emission, metrological applications are less demanding, the actual sources of uncertainty that arise when calibrating detectors using the photon pairs produced in PDC are several. The most critical relate to the process of separating the efficiency of the detector to be calibrated from the efficiency of the detection channel taken as a whole (including of course the mode matching). In simple terms, if the trigger detector senses a photon and the detector to be calibrated does not detect its twin then we do not know whether the photon was actually incident on the detector or was lost upstream in the detection channel. To minimize this uncertainty, a specific design of the system to maximize the collection of the photons correlated to those seen by the trigger detector, and eventually a careful measurement or calculation of any residual collection losses that cannot be designed out of the system, are mandatory. The uncertainty of this residual loss contributes, in large part, to the ultimate limit of the uncertainty of the final detector efficiency.

Collection system losses can be classified as either conventional transmittance losses due to reflection and absorption, or geometric losses due to causes such as limiting apertures, finite detector area, or positioning errors.

Transmittance losses may be handled straightforwardly; the transmittance of optical components can be measured conventionally with high accuracy, or in some cases the losses can be calculated with good results (e.g. the losses in the down-conversion crystal).

Geometric losses due to positioning errors can also be characterized in a clear-cut manner.

A more challenging loss to quantify occurs because there is a spread of emission directions and positions for the photons correlated to those seen by the trigger detector. Because different wavelengths of down-conversion have different emission angles, the bandwidth seen by the trigger affects the angular spread of the light that must be coupled onto the detector under test, whose angular acceptance and frequency-selective elements have to be large enough to accept all frequencies correlated to photons arriving at the trigger detector. In this respect the analysis of the angular and spectral spread of the photons described in the previous sections is of paramount importance in estimating the final accuracy of the source.

The collection losses can be quantified and experimentally verified, but there are a number of other systematic effects related to the detectors. As an example, 
detector after-pulsing can affect efficiency measurements; also, threshold setting, dead time, nonlinearities (both reversible and permanent), spatial response non-uniformity of the detector, and other coincidence measurement related issues. An example on how deadtime, background and after-pulsing of photon-counting detectors affect high accuracy in detector calibration is reported in [80]. Here a method for separating the correlated signal from the background signal, and considerations of pulse timing and after-pulsing issues and how these effects should be considered in detector calibration, are presented.

Recently [81] the bi-photon field has been employed in a self-calibration scheme where the recombination of two temporally delayed single photon beams from PDC is used to measure the single photon detection efficiency of one detector, without even the need of a trigger detector. This technique reduces some of the uncertainty associated with the two detector scheme of the heralding configuration (e.g. geometrical/spectral collection and positioning), but some of the timing-associated problems as well as optical losses can still remain and can be even more problematic.

In reference [82] this primary standard method to calibrate detection efficiency has been verified and its uncertainty compared to a substitution method using a conventionally calibrated transfer detector tied to a national primary standard detector scale. The relative standard uncertainty for the correlated-photon method of $0.18 \%$ is comparable with the substitution method of $0.17 \%$. This is the highest accuracy characterization and independent verification of the correlated-photon method yet achieved. Even if the conventional radiometry can provide similar uncertainty as the photon counting technique, this last is still of interest because it is by far easier and less expensive to implement, as well as direct, and it can be performed in any laboratory without maintaining expensive primary radiometric standard and related dissemination scale. However, efforts are still worthy in the direction of improving the final uncertainty in PDC-based methods aiming realistically at one order of magnitude better. To improve the uncertainty of the primary two-photon beam method, more efficient sources of correlated photons are required and faster detection systems to link the photon counting directly to the analogue regime of optical power standards. In terms of conventional radiometry, a microwatt cryogenic radiometer is presently under development [18], with a next-step project of a photon flux cryogenic radiometer undergoing measurement of photon flux of $10^{10} \mathrm{~s}^{-1}$, taking advantage of developments in superconducting technology. On the side of sources, a possible generator of correlated photons comes from the development of $\chi^{(3)}$ single-mode photonic fiber-based sources with pair rates up to $10^{7} \mathrm{~s}^{-1}$, with very low input pump power $[16,17]$. Finally to improve the possible measurable rate in the photon counting regime, a scheme made of a pool of photon-counting detectors operating as a unit could be used $[83,84]$ with better accuracy. The proposed scheme consists of a 1-by- $N$ optical switch that takes a single input stream of photons and distributes them to members of an array of $N$ detectors. A switch controller monitors which detectors have fired recently and are thus dead, and then routes subsequent incoming pulses to a detector that is ready. An active switching scheme is in fact preferable to passive schemes (using passive beamsplitter trees and detector arrays), and also to the performance of a single detector with much reduced deadtime, even in cases where the switching time is not negligible. A modeling of this scheme shows that switch transition times are negligible when they are less than $2 \%$ of the individual detector deadtimes.

\section{Conclusions}

We have summarized some of the remarkable results obtained in PDC production for heralding single photons. The two main problems of heralding single photons for different applications have been addressed, and possible solutions proposed.

The first group of solutions reduce the randomness of the pair generation for a truly on-demand source as well as the problem of having true single photons. While the multi-pair production is more easily tailored and controlled by increasing the number of down-conversions within the same crystal, the pseudo-deterministic production of single photons is still a problem, because of the losses in photon storage systems and in optical switches. The second group of solutions deals with preparing single photons in definite spectral and spatial modes and maintaining high efficiency of pair production. A review of achievements in PDC realization with focused pump beam and of single and multi-mode collection of photon pairs has been proposed in several configurations of CW and pulsed pumps. In particular, a short-pulsed and strongly focused pump produce an entanglement between spectral and transverse photon modes. Single mode collection is preferable to multi-mode in terms of source efficiency, when the modes of pump and photons are properly matched, which implies that the pump must be focused inside the crystal. Conversely, a collimated pump is more suited for multi-mode collection and preparation, even if in this case the final efficiency of the source, in terms of collected pairs, is lower. Therefore a waveguide solution is more promising than bulk crystals for heralded photon sources, providing a better final efficiency in terms of mode matching and pair production. QKD applications of heralded single photon sources require also a telecom wavelength operation, which is better implemented in the heralding configuration using non degenerate PDC, in order to have the heralding photon in the visible, where detectors are more efficient. Finally a summary of the state of the art in quantum communication and radiometry applications is provided.

\section{References}

1. A. Migdall, J. Dowling, J. Mod. Opt. 51, 1265 (2004)

2. P. Kumar, P. Kwiat, A. Migdall, S. Nam, J. Vuckovic, F.N.C. Wong, Quantum Inf. Processing 3, 215 (2004) 
3. C.H. Bennett, G. Brassard, Proc. IEEE Int. Conf. Computers, Systems, and Signal Process. 175 (1984)

4. N. Gisin, G. Ribordy, W. Tittel, H. Zbinden, Rev. Mod. Phys. 74, 145 (2002)

5. B. Temelkuran, S.D. Hart, G. Benoit, J.D. Joannopoulos, Y. Fink, Nature, 650 (2002)

6. T. Curcic, M.E. Filipkowski, P.A. D'Ambrosio, S.A. Wolf, M. Foster, D. Cochran, ACM SIGCOMM Comp. Communs. Rev. 34, 1 (2004)

7. A. Lacaita, F. Zappa, S. Cova, P. Lovati, Appl. Opt. 35, 2986 (1996)

8. D. Rosenberg, A.E. Lita, A.J. Miller, S. Nam, Noise-free high-efficiency photon-number-resolving detectors. e-print arXiv:quant-ph/0506175 (2005)

9. S. Cova, M. Ghioni, A. Lotito, I. Rech, F. Zappa, J. Mod. Opt. 51, 1267 (2004)

10. A. Rochas, P. Besse, R. Popovic, Actively recharged single photon counting avalanche CMOS photodiode with less than 9 ns dead time, in Proceedings of The 16th European Conference on Solid-State Transducers (Prague, Czech Republic, 2002)

11. K. Sanaka, K. Kawahara, T. Kunga, Phys. Rev. Lett. 86 , $5620(2001)$

12. K. Banaszek, A.B. U'Ren, I.A. Walmsley, Opt. Lett. 26 , $1367(2001)$

13. A. U'Ren, C. Silberhorn, K. Banaszek, I. Walmsley, Phys. Rev. Lett. 93, 093601 (2004)

14. X. Li, P.L. Voss, J.E. Sharping, P. Kumar, Phys. Rev. Lett. 94, 053601 (2005)

15. J.G. Rarity, J. Fulconis, J. Duligall, W.J. Wadsworth, P.S.J. Russell, Opt. Exp. 13, 534 (2005)

16. J. Fulconis, O. Alibart, W.J. Wadsworth, P.S.J. Russell, J.G. Rarity, Opt. Exp. 13, 7572 (2005)

17. J. Fan, A. Migdall, Opt. Exp. 13 (2005)

18. J. Cheung, C.J. Chunnilal, E.R. Woolliams, N.P. Fox, J.R. Mountford, J. Wang, P.J. Thomas, J. Modern Opt. 54, 373 (2007)

19. P. Finocchiaro et al., J. Modern Opt. 54, 199 (2007)

20. A. Giudice et al., J. Modern Opt. 54, 225 (2007)

21. B. Aull, A. Loomis, D. Young, R. Heinrichs, B. Felton, P. Daniels, D. Landers, Lincoln Lab. J. 13, 335 (2002)

22. S. Tudisco, Sinphos: a single photon spectrometer for biomedical applications, in European Conference on Biomedical Optics (2005)

23. P. Grangier et al., New J. Phys. 6 (2005)

24. P. Grangier, I. Abram, Phys. World 16, 31 (2003)

25. D.N. Klyshko, Photons and Nonlinear Optics (Gordon and Breach Science Publishers, 1988)

26. A. Migdall, Phys. Today 52, 41 (1999)

27. A.L. Migdall, S. Castelletto, I.P. Degiovanni, M.L. Rastello, Appl. Opt. 41, 2914 (2002)

28. W. Tittel, J. Brendel, H. Zbinden, N. Gisin, Phys. Rev. Lett. 84, 4737 (2000)

29. E. Knill, R. Laflamme, G.J. Milburn, Nature 409, 46 (2001)

30. V. Giovannetti, S. Lloyd, L. Maccone, Phys. Rev. A 65 , 022309 (2002)

31. D.V. Strekalov, A.V. Sergienko, D.N. Klyskho, Y.H. Shih, Phys. Rev. Lett. 74, 3600 (1995)

32. A.N. Boto et al., Phys. Rev. Lett. 85, 2733 (2000)

33. S.L. Braunstein, A.K. Pati, Quantum Information Theory with Continuous Variables (Kluwer, 2002)
34. M. Bondani, A. Allevi, G. Zambra, M.G.A. Paris, A. Andreoni, Phys. Rev. A 76, 013833 (2007)

35. T.B. Pittman, B.C. Jacobs, J.D. Franson, Phys. Rev. A 66, $042303(2002)$

36. A. Migdall, D. Branning, S. Castelletto, Phys. Rev. A 66, $053805(2002)$

37. N.A. Peters, K.J. Arnold, A.P. VanDevender, E.R. Jeffrey, R. Rangarajan, O. Hosten, J.T. Barreiro, J.B. Altepeter, P.G. Kwiat, Towards a quasi-deterministic single-photon source, in Proceedings of The SPIE Conference Quantum Communications and Quantum Imaging IV OEI122, Optics and Photonics (San Diego, 2006)

38. C. Kurtsiefer, M. Oberparlieter, H. Weinfurter, Phys. Rev. A 64, 023802 (2001)

39. F.A. Bovino, P. Varisco, A.M. Colla, G. Castagnoli, G. Di Giuseppe, A.V. Sergienko, Opt. Commun. 227, 343 (2003)

40. S. Castelletto, I.P. Degiovanni, M. Ware, A. Migdall, Coupling efficiencies in single photon on-demand sources, in SPIE Conf on Quantum Communications and Quantum Imaging, San Diego, CA, (2003) 5161, 48

41. T.B. Pittmann, B.C. Jacobs, J.D. Franson, Opt. Commun. 246, 545 (2005)

42. A. Dragan, Phys. Rev. A 70, 053814 (2004)

43. R. Andrews, E.R. Pike, S. Sarker, Opt. Exp. 12, 3264 (2004)

44. S. Castelletto, I.P. Degiovanni, V. Schettini, A. Migdall, Opt. Express 13, 6709 (2005)

45. D. Ljunggren, M. Tengner, Phys. Rev. A 72, 062301 (2005)

46. M. Pelton, P. Marsden, M. Tengner D. Ljunggren, A. Karlsson, Opt. Express 12, 3573 (2004)

47. M.A. Albota, E. Dauler, J. Modern Opt. 51, 1417 (2004)

48. C.E. Kuklevicz, M. Fiorentino, G. Messin, Franco N.C. Wong, J.H. Shapiro, Phys. Rev. A 69, 041801 (2004)

49. S. Castelletto, I.P. Degiovanni, V. Schettini, A. Migdall, Metrologia 43, S56 (2006)

50. J. Soderholm, K. Hirano, S. Mori, S. Inoue, Analysis of the generation of photon pairs in periodically poled lithium niobate, in Proceedings of the 8th International Symposium on Foundations of Quantum Mechanics in the Light of New Technology (World Scientific, 2006), pp. 46-49

51. O. Alibart, S. Tanzilli, D.B. Ostrowsky, P. Baldi, Elec. Lett. 30 (2001)

52. A. Trifonov, A. Zavriyev, V. Denchev, A. Leverrier, J. Modern Opt. 54, 305 (2007)

53. S. Mori, J. Soderholm, N. Namekata, S. Inoue, Opt. Commun. 264, 156 (2006)

54. O. Alibart, S. Tanzilli, D.B. Ostrowsky, P. Baldi, Opt. Lett. 30, 1539 (2005)

55. I. Avrutsky, A.V. Sergienko, Phys. Rev. A 71, 033812 (2005)

56. A.B. U'Ren, C. Silberhorn, R. Erdmann, K. Banaszek, W.P. Grice, I.A. Walmsley, M.G. Raymer, Generation of pure-state single-photon wavepackets by conditional preparation based on spontaneous parametric downconversion. e-print ArXiv:quant-ph/0611019 (2006)

57. A. Migdall, D. Branning, S. Castelletto, A single photon on-demand source with decoupled single and multiphoton probabilities, in Proc. 6th Int'l Conference on Quantum Communications Measurement and Computing, July 2226, 2002, Cambridge MA, edited by J. Shapiro, O. Hirota (Rinton Press, 2003), pp. 528-531

58. S. Fasel, New J. Phys. 6, 163 (2004)

59. C.K. Hong, L. Mandel, Phys. Rev. Lett. 56, 58 (1986) 
60. A.I. Lvovsky, H. Hansen, T. Aichele, O. Benson, J. Mlynek, S. Schiller, Phys. Rev. Lett. 87, 050402 (2001)

61. M. Zukowski, A. Zeilinger, H. Weinfurter, Ann. N.Y. Acad. Sci. 755, 91 (1995)

62. D. Bouwmeester, J.W. Pan, K. Mattle, M. Eibl, H. Weinfurter, A. Zeilinger, Nature 390, 575 (1997)

63. M.H. Rubin, Phys. Rev. A 54, 5349 (1996)

64. D.N. Klyskho, Phys. Lett. A 132, 299 (1988)

65. S. Castelletto, I.P. Degiovanni, M. Ware, A. Migdall, New J. Phys. 6, 87 (2004)

66. S. Castelletto, I.P. Degiovanni, G.P. Furno, A. Migdall, V. Schettini, M. Ware, IEEE Trans. Inst. Meas. 54, 890 (2005)

67. R.S. Bennik, Y. Liu, D.D. Earl, W. Grice, Phys. Rev. A 74, $023802(2006)$

68. N. Boeuf, D. Branning, I. Chaperota, E. Dauler, S. Guerin, G. Jaegera, A. Muller, A. Migdall, Opt. Eng. 39, 1016 (2000)

69. O. Alibart, S. Tanzilli, D.B. Ostrowsky, P. Baldi, High-performance heralded single-photon source. e-print arXiv:quant-ph/0405075 (2004)

70. A. Soujaeff, S. Takeuchi, K. Sasaki, T. Hasegawa, M. Matsui, J. Modern Opt. 54, 467 (2007)

71. S. Castelletto, I.P. Degiovanni, V. Schettini, T. Del Rosso, G. Margheri, L. Papi, S. Sottini, JOSA B 25, 7 (2007)
72. T.E. Keller, M.H. Rubi, Phys. Rev. A 56, 1534 (1997)

73. J. Fan, A. Migdall, L. Wang, A twin photon source, OPN March 2007 (2007)

74. J. Fulconis, O. Alibart, J.L. O Brien, W.J. Wadsworth, J.G. Rarity, Photonic crystal fibre source of photon pairs for quantum information processing. e-print arXiv:quant-ph/0611232 (2006)

75. D.N. Klyshko, Sov. J. Quantum Electron. 10, 1112 (1980)

76. A. Migdall, R. Datla, A. Sergienko, J.S. Orszak, Y. Shih, Metrologia 32, 479 (1995)

77. G. Brida, S. Castelletto, C. Novero, M.L. Rastello, Metrologia 35, 397 (1998)

78. A. Migdall, Phys. Today 52, 41 (1999)

79. M. Ware, A. Migdall, J. Modern Opt. 51, 1549 (2004)

80. M. Ware, A. Migdall, J.C. Bienfang, S.V. Polyakov, J. Mod. Opt. 54, 361 (2007)

81. X.-H. Chen, Y.-H. Zhai, D. Zhang, L.-A. Wu, Opt. Lett. 31, 2441 (2006)

82. S.V. Polyakov, A.L. Migdall, Opt. Exp. 15, 1390 (2007)

83. S. Castelletto, I.P. Degiovanni, V. Schettini, A. Migdall, J. Modern Opt. 54, 337 (2007)

84. S. Castelletto, I.P. Degiovanni, S. Polyakov, V. Schettini, A. Migdall, Achieving higher photon counting rates using multiplexed detectors, in SPIE Quantum Communications and Quantum Imaging IV, (2006) 6305, 63050R 